
allemande

47-2 | 2015

Retour sur le modèle Rhénan : humanisme, capitalisme et métropolisation | Patrimonialisation du passé « allemand » en Europe centrale après 1990

\title{
Restaurer la sensibilité au paysage
}

Deux mouvements de patrimonialisation aux confins de la Bohême pendant la seconde moitié $\mathrm{du} \mathrm{xx}^{\mathrm{e}}$ siècle

\section{Ségolène Plyer}

\section{(2) OpenEdition}

Édition électronique

URL : https://journals.openedition.org/allemagne/309

DOI : 10.4000/allemagne.309

ISSN : 2605-7913

Éditeur

Société d'études allemandes

Édition imprimée

Date de publication : 16 décembre 2015

Pagination : 439-456

ISSN : 0035-0974

Référence électronique

Ségolène Plyer, «Restaurer la sensibilité au paysage », Revue d'Allemagne et des pays de langue allemande [En ligne], 47-2 | 2015, mis en ligne le 13 décembre 2017, consulté le 25 mai 2021. URL http://journals.openedition.org/allemagne/309; DOI : https://doi.org/10.4000/allemagne.309 


\section{Restaurer la sensibilité au paysage Deux mouvements de patrimonialisation aux confins de la Bohême pendant la seconde moitié du Xxe siècle}

- Ségolène Plyer *

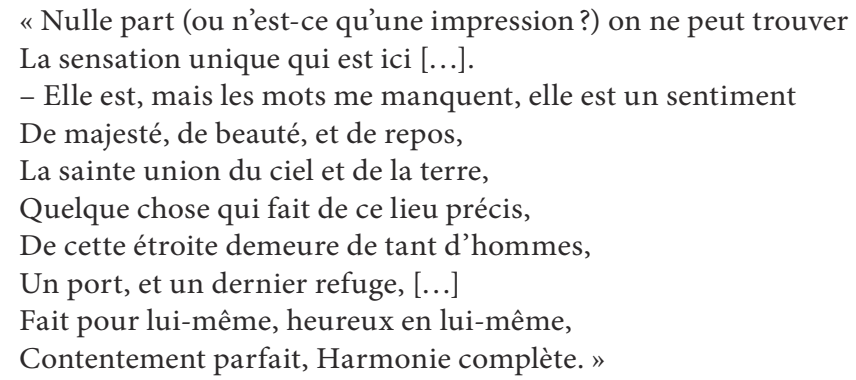

Qui a jamais lu de publication faite par et pour les expulsés allemands d'Europe de l'Est après 1945 pourrait aisément croire ce poème extrait de l'une d'entre elles, qui déplorerait le sentiment de plénitude à jamais perdu avec la patrie d'origine. Il s'agit pourtant d'une œuvre écrite dans des circonstances bien différentes par William Wordsworth (1770-1850), chantre de la région des Lacs à l'est des Midlands alors que l'industrialisation commençait de marquer profondément la campagne anglaise ${ }^{(1)}$. Sa conception sentimentale du paysage n'est, paradoxalement, pas sans intérêt pour comprendre les mouvements de patrimonialisation qu'on peut observer aux confins de la Bohême dont les habitants furent massivement déplacés (souvent de force) dans les années suivant la Seconde Guerre mondiale. Elle nous servira de fil conducteur pour analyser les efforts de "patrimonialisation » dans cette région, c'est-à-dire l'ensemble des pratiques voulant faire revivre le passé, le conserver et le transmettre. Ces derniers

* Maître de conférences, Université de Strasbourg.

1 William Wordsworth, "Home at Grasmere » (poème écrit entre 1800 et 1806), cité et traduit par Charles-François Mathis, in: In Nature We Trust. Les paysages anglais à l'ère industrielle, Paris, Presses de l'Université Paris-Sorbonne, 2010, p. 94. 
se portent d'abord sur le paysage qui représente pour nombre d'acteurs locaux le patrimoine par excellence, c'est-à-dire un ensemble exceptionnel digne d'être préservé.

Curieusement, l'étude de la démarche actuelle permet d'en découvrir une autre passée jusqu'alors inaperçue pour la recherche, la patrimonialisation par le souvenir effectuée par les expulsés allemands dans leurs périodiques après 1945. Ces publications formeront l'essentiel de notre corpus, que nous présenterons avant de comparer la patrimonialisation d'aujourd'hui à celle de l'immédiat après-guerre, pour mettre en lumière l'importance du paysage dans des entreprises de régénération comparables, malgré les différences de contexte et d'acteurs.

\section{Recueillir le patrimoine mémoriel allemand}

Notre terrain sera l'extrémité orientale des Monts des Sudètes et les bassins qui jalonnent son inclinaison vers la Moravie, région de frontière entre la Bohême et la Silésie, parce que nous connaissons assez bien cette région par d'autres recherches et que les districts de Trutnov (Trautenau en allemand) et Broumov/Braunau formèrent une relative unité culturelle tandis qu'aujourd'hui, l'originalité des initiatives prises par certains habitants tchèques depuis 1990 pour restaurer ces régions considérées comme sinistrées justifie de les prendre en exemple.

En effet, tant par la langue que par les liens entre eux et la frontière silésienne, les deux districts administratifs qui nous occupent s'individualisaient assez clairement par rapport au reste de la province de Königingrätz/Hradec Kralové dont ils faisaient partie: majoritairement peuplés de germanophones, ils se trouvaient au bout des Monts des Géants (Riesengebirge/Krkonoše) et partageaient la même structure socio-économique d'agriculture mixte (mêlant élevage et polyculture, assez prospère en plaine, mais pauvre en montagne où l'industrie domestique et le tourisme étaient d'indispensables compléments de revenu) et d'industrie, majoritairement textile, qui avait pris le relais du filage et du tissage à domicile dans la seconde moitié du XIX siècle. En 1929, Trutnov/Trautenau comptait 14584 habitants dont 2791 tchécophones, soit $20 \%$, l'industrialisation y ayant amené une immigration parlant tchèque entre 1850 et 1870; Broumov/Braunau, où l'industrie, plus tardive et mécanisée, n'avait pas modifié le caractère d'abord germanophone de la population, comptait alors 6818 habitants, dont 867 Tchèques $^{(2)}$.

Dans l'entre-deux-guerres, la région connut une crise latente due aux difficultés du textile, qui se traduisit par de l'exode rural et une situation sanitaire dégradée. Le chômage des années trente frappa durement ces districts exportateurs, et fit le succès du parti autonomiste de Konrad Henlein qui réunit $60 \%$ des votes aux élections municipales de 1935. Ce dernier prit une orientation irrédentiste en 1937 et se fit l'instrument de l'annexion de la région sudète par le Troisième Reich en 1938. En mai 1945, l'expulsion de la population germanophone commença; quasiment achevée en octobre 1946, elle mit fin à plus de six siècles de présence allemande dans la région. Depuis la fin de la Guerre froide et l'effondrement du Bloc de l'Est, la région souffre d'un fort taux de chômage. Le tourisme de proximité, tchèque et polonais, est devenu une ressource non

2 Chiffres tirés de l'annuaire Místopisný slovník československé Republiky de 1929. À titre de comparaison, Náchod, la troisième ville de la région que nous n’étudierons pas ici puisque sa population germanophone ne fut pas suffisante pour donner naissance à une publication spécifique après la guerre, comptait à la même époque 12244 habitants, dont 11954 se disaient tchèques. 
négligeable qui fait des curiosités locales (le parc naturel des Krkonoše et les monuments, notamment l'ensemble baroque du pays de Broumov) des enjeux économiques.

\section{Les bulletins d'expulsés}

1945 fut donc une année de rupture qui rendit centrale la question du patrimoine dans les relations germano-tchèques. Nous allons l'aborder tout d'abord dans les publications des expulsés (avant d'étudier les parutions tchèques dans notre seconde partie). En janvier 1948 parut en Bavière la première «Circulaire » bimensuelle du pays de Braunau (Braunauer Rundbrief ou $B R$ ), tandis que plusieurs titres pour le district de Trautenau avaient commencé d'être tirés depuis 1946 dans le sud de l'Allemagne de l'Ouest, souvent irrégulièrement, avant d'être unifiés par le mensuel Riesengebirgsheimat ("La patrie des Monts des Géants », en abrégé RGH) entre 1949 et 1958. L'Institut für Volkskunde der Deutschen in Europa (Institut d'ethnologie des Allemands d'Europe) conserve à Fribourg-en-Brisgau une collection presque complète des deux séries ${ }^{(3)}$. Jusqu'au début des années soixante-dix, l'activité de ces périodiques fut vraisemblablement suffisante pour qu'un "livre de la patrie " (Heimatbuch), comme il s'en écrivait depuis les années cinquante dans d'autres groupes d'expulsés installés en RFA, soit considéré comme superflu. En même temps, ces publications ont réussi à s'imposer comme les organes de l'ancienne patrie, puisque depuis leur création, aucune publication indépendante n'a été recensée ${ }^{(4)}$.

Le patrimoine mémoriel des anciens habitants de Bohême orientale se constitua autour de trois types d'articles. En nombre de pages, c'est tout d'abord l'histoire des institutions, des associations et des clubs sportifs qui s'imposa. Suivirent des descriptions détaillées du paysage de la Heimat, soit sous forme d'inventaire de ses richesses naturelles et historiques, soit sous la forme d'itinéraires de promenade. De fait, l'intérêt pour la patrie d'origine ne se démentit jamais; la $R G H$ et le $B R$ eurent à cœur de garder le lien avec «l'ancienne patrie » (alte Heimat) jusqu'à aujourd'hui. Dans les années quarante, la rubrique qui lui était consacrée pouvait remplir jusqu'à deux des premières pages, alimentée avant tout par les nouvelles d'anciennes connaissances restées sur place, la traduction d'avis et de bulletins municipaux, enfin le récit des voyageurs à leur retour en RFA. Cet intérêt se portait d'abord sur le devenir des anciens voisins, mais aussi des maisons et fermes, ainsi que des administrations (poste, hôpital); l'attention au bâti étant sans doute favorisée par une conception ancienne d'héritage à maison, l'idée que la maison est un dépôt des ancêtres à conserver sans modification, avec une piété particulière ${ }^{(5)}$.

3 Avec quelques lacunes pour Braunau en ce qui concerne les tout premiers numéros, qui peuvent être trouvés à la Bibliothèque nationale de Prague et dans les archives du Heimatkreis Braunau/ Sudetenland (Association des anciens habitants de Braunau) à Forchheim.

4 D’après Rudolf Hemmerle, Heimat im Buch: sudetendeutsche Heimatbücher, Ortsmonographien, Karten, Heimatblätter, Heimatzeitschriften, Jahrbücher und Kalendar nach 1945. Eine Bibliographie,

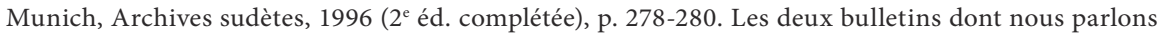
existent toujours, avec en complément des sites Internet gérés par des membres des mêmes associations.

5 Sur ce point, nous nous permettons de renvoyer à notre thèse de doctorat («Les Allemands des Sudètes et l'Allemagne. L'exemple de Braunau en Bohême », Paris I, 2007), t. I, p. 84-88, « Le Heimatrecht et le système d'héritage paysan ». 
Enfin, les témoignages sur le passé apparaissent avec la même fréquence que les rubriques précédentes. Dans les premiers numéros, que nous avons étudiés en détail, ces souvenirs portèrent, dans la $R G H$, surtout sur l'expulsion et ses vicissitudes, tandis que ceux du $B R$ dressaient le portrait des personnalités locales, recueillaient les récits à caractère historique des plus anciens et n'évoquaient guère l'expulsion. Cette orientation continua de distinguer les publications de Braunau jusqu'à nos jours; il faut certainement y voir l'influence des fondateurs de la revue, les bénédictins de Braunau, installés à Rohr en Bavière où ils ouvrirent un lycée. Ils entreprirent notamment de collecter le plus tôt possible les témoignages des vieillards dont on pouvait craindre la disparition rapide, ce qui fait des premières années du bulletin une riche collection de récits sur des événements remontant parfois jusqu'aux années 1840, source originale sur la société locale des notables et des paysans aisés, puisque c'étaient ces derniers qui étaient interrogés - la valeur sociale de l'enquêté faisant, pour les rédacteurs du $B R$, celle de son témoignage. Au fil du temps, les notables s'effacèrent; les biographies se figèrent, dans le sens qu'il était rare qu'un nouveau matériau vînt enrichir la collection déjà présente, qu'on ne fit plus que reprendre en y ajoutant toutefois le portrait des responsables du Heimatkreis de Braunau (Association des anciens habitants allemands) et, parfois, quelque nouvelle personnalité qui pouvait avoir été introduite par une redécouverte, due par exemple à l'intérêt " pour les concitoyens juifs » au milieu des années 1960 ou aux personnalités locales par les autorités tchèques ces dernières années (ainsi du compositeur Joseph Tichatschek, né en 1807 à Ober-Weckelsdorf/Horní Teplice).

\section{Les Heimatbücher}

Dans les années soixante seulement furent aussi publiés par souscription des Heimatbücher dont la traduction par " livres d'histoire locale " ne rend pas entièrement compte de la fonction: selon les critères de Wolfgang Kessler ${ }^{(6)}$, il s'agit d'ouvrages d'une certaine tenue, mais d'une lecture facile, abordant tous les aspects nécessaires pour connaître la petite patrie qu'est la Heimat (géographie, botanique, zoologie, économie, démographie, histoire, ethnographie et folklore...), privilégiant le détail des faits et les chroniques sur la mise en contexte historique, achetés par tous, mais condamnés à une péremption assez rapide par leur absence de pertinence scientifique, puisqu'ils sont rédigés par des non-spécialistes et se bornent aux sources disponibles dont, au premier chef, les souvenirs de la société locale dont ils font le portrait. Néanmoins, les années soixante représentent une phase de production plus scientifique ${ }^{(7)}$ : c'est le moment où les Heimatbücher, caractérisés depuis le début du $\mathrm{XX}^{\mathrm{e}}$ siècle par leur éloignement croissant des sciences historiques, s'efforcèrent de combler le fossé entre elles et le récit grand public qui les caractérise, par le recours à des documents authentiques et des contributions satisfaisant aux critères de la recherche.

6 Wolfgang KessleR, « Ostdeutsche, sudetendeutsche und südostdeutsche Heimatbücher - Erinnerung und Dokumentation ", in: Fondation Ostdeutscher Kulturrat (éd.), Ost- und südostdeutsche Heimatbücher und Ortsmonographien nach 1945. Eine Bibliographie zur historischen Landeskunde der Vertreibungsgebiete. Cette introduction à une bibliographie fondamentale est citée par Matthias Beer dans « Das Heimatbuch als Schriftenklasse. Forschungsstand, historicher Kontext, Merkmal und Funktionen ", in: M. BeER (dir.), Das Heimatbuch. Geschichte, Methodik, Wirkung, Göttingen, Vandenhoeck \& Ruprecht unipress, 2010, p. 9-39, ici p. 16-17.

7 M. BeER, Das Heimatbuch (note 6), p. 34. 
Les Heimatbücher qui nous intéressent puisèrent essentiellement aux collections constituées par les expulsés $^{(8)}$ (comme le musée de Braunau à Forchheim) et aux publications du premier mouvement de patrimonialisation, celui des années 18901910 qui avait servi d'abord aux besoins de l'enseignement primaire $^{(9)}$; ainsi que, parfois, à quelques publications spécialisées - trop pour replacer méthodiquement les nombreux détails historiques, dont ces travaux sont friands, dans un contexte plus général. Ces ouvrages ${ }^{(10)}$ furent rédigés par des personnes reconnues pour leur savoir par les anciens habitants, mais rarement des historiens. Conçus pour être maniables et représentatifs plutôt qu'exhaustifs, ils ne purent reprendre dans leur intégralité les informations glanées au cours des décennies par les périodiques, ce qui conserve à ces derniers leur intérêt documentaire.

Le monopole de fait des publications par les Heimatkreise (sur papier et maintenant par Internet) montre que l'héritage mémoriel qu'ils ont sauvegardé et constitué en patrimoine a été accepté, reçu, tout au moins non mis en question par les personnes se sentant attachées à la même origine. La raison en est sans doute que cette constitution s'est toujours faite en étroite relation avec la mémoire communicationnelle grâce à des rencontres régulières des anciens habitants organisées par les Heimatkreise, ainsi que par consensus autour de la formulation des récits, notamment par la référence aux mêmes auteurs littéraires (comme le Silésien Gerhard Hauptmann, considéré comme une autorité sur la vie des tisserands à domicile y compris dans les Monts des Géants). De fait, la $R G H$ et le $B R$ choisirent moins entre mémoires individuelle, communicationnelle, collective et savoir scientifique qu'ils ne les mêlèrent au sein d'un même numéro, tout en distinguant clairement les niveaux de légitimité - le $B R$ étant le plus systématique, avec un cahier de Heimatkunde ("information sur la patrie d'origine ») au centre de la revue, imprimé sur papier jaune et pouvant être considéré comme une publication à part, où des travaux scientifiques intéressant la région sont recensés. Avec la réserve que le filtre n'est pas scientifique (accueillant de façon neutre tout

8 Signalons à ce sujet la thèse récente de Cornelia Eisler, Verwaltete Erinnerung - symbolische Politik. Die Heimatsammlungen der deutschen Flüchtlinge, Vertriebenen und Aussiedler, Oldenburg, De Gruyter/Oldenbourg (Schriften des Bundesinstituts für Kultur und Geschichte der Deutschen im östlichen Europa, 57), 2015.

9 Il s'agit, par les Associations des professeurs et instituteurs du district scolaire de Braunau, de la Braunauer Heimatkunde, Braunau en Bohême (Johannes Swirak), 1894; pour Trautenau, de Josef Demuth, Der politische Bezirk Trautenau, Éditions de l'association des instituteurs du district de Trautenau, 1910, ainsi que de la revue d'histoire régionale de l'entre-deux-guerres Das Riesengebirge in Wort und Bild (d’après Vladimír Wolf, " Nástin historie dějepisectví Trutnovska 20. století " [Esquisse d'une historiographie de la région de Trutnov au $\mathrm{xx}^{\mathrm{e}}$ siècle], Krkonoše-Podkrkonoší, Supplementum, 8 [2008], p. 11). Ce sont, en effet, les "leçons de chose " fondées sur l'histoire, la géographie et les conditions locales (Heimatkunde), introduites dans l'enseignement sous la lointaine influence de Pestalozzi, qui poussèrent à rédiger des Heimatbücher (M. BEER, «Das Heimatbuch als Schriftenklasse ", in: Das Heimatbuch [note 6], p. 28-29).

10 Ils sont peu nombreux dans le district de Trautenau (le premier, sur Schatzlar, parut en 1993 et n'eut longtemps pas de postérité; celui de Trautenau même date seulement de l'an 2000). En revanche, le Heimatkreis Braunau mit en œuvre un programme de documentation systématique de tous les villages germanophones, après que le Heimatbuch Braunau fut publié en 1971 sous la direction du professeur d'histoire Hugo Herrmann. Les Heimatbücher (ou Dorfbücher) du pays de Braunau furent rédigés entre 1982 et 2003 et sont disponibles dans plusieurs grandes bibliothèques d'Europe centrale. 
travail répondant aux exigences des sciences historiques), mais politique (conforme aux vues de la Landsmannschaft, notamment en ce qui concerne l'histoire contemporaine $\left.{ }^{(11)}\right)$, il y a eu là matière à constituer un patrimoine immatériel. Ce dernier a-t-il servi aux habitants actuels du pays?

\section{Transmettre, préserver, faire vivre le passé aujourd'hui}

\section{Reconstituer ensemble la mémoire des confins?}

Les réflexions tchèques sur le patrimoine datent d'après la "Révolution de velours » de l'hiver 1989 et, dans les régions qui nous intéressent, sont nées de la situation particulière des confins jadis peuplés en majorité par des germanophones. En 1945, la restauration de la Tchécoslovaquie dans son intégrité territoriale de 1938 avait été conçue par les nouvelles autorités comme une révolution qui instaurerait un État à tendance socialiste, intérieurement uni et appuyé sur la puissance de l'URSS. Dans les régions germanophones, le flottement du pouvoir parallèlement à l'expulsion des germanophones fut propice à des actes de violence contre les personnes, de brigandage et de destruction des objets culturels liés à la présence allemande, dans une atmosphère parfois iconoclaste ${ }^{(12)}$; une partie des livres de la bibliothèque bénédictine de Broumov fut ainsi envoyée à la décharge par bennes entières ${ }^{(13)}$. Les autorités eurent de la peine à repeupler le pays. En 1952, la densité des régions frontalières était de 84 habitants au kilomètre carré, contre 127 en $1930^{(14)}$, ce qui entraîna l'abandon des exploitations agricoles isolées et des grands bâtiments difficiles à entretenir, qui tombèrent en ruine.

Après 1989, les changements économiques s'accélérant, la décrépitude des confins et ce qui était ressenti comme une stagnation générale due à l'appauvrissement culturel et à l'absence de perspective des habitants, atteignirent un niveau préoccupant dans certaines régions. La plupart des acteurs locaux tchèques, notamment les maires, attendirent des anciens habitants allemands la transmission de «l'héritage culturel de nos prédécesseurs et une aide pour le perpétuer ${ }^{(15)}$ et ce tout particulièrement dans la région de Broumov, très à l'écart du reste du pays. Après la Révolution de velours, les Heimatbücher, avec leur abondante illustration de cartes postales anciennes, leurs anecdotes sur les lieux et les légendes locales, y furent vendus avec succès même sans

11 On trouvera une approche de l'historiographie de la Sudetendeutsche Landsmannschaft (SL) chez Tobias WegER, Volkstumskampf ohne Ende? Sudetendeutsche Organisationen, 1945-1955, Francfort/ Berlin/Berne, Peter Lang, 2008, qui consiste, dans ses aspects les moins défendables, à rejeter la détérioration des relations entre germanophones et tchécophones sur ces derniers, notamment sur le président Edvard Beneš; et à taire toute participation d'Allemands des Sudètes à la montée du nazisme (en Allemagne comme en Tchécoslovaquie) puis au III Reich (p. 323-358).

12 Tomáš StanĚK, Poválečné 'excesy'v českých zemích v roce 1945 a jejich vyšetrování (Les " excès " commis entre mai et décembre 1945 dans les Pays tchèques et les enquêtes judiciaires auxquels ils ont donné lieu), Prague, Ústav pro soudobé dějiny Akademie věd České republiky, 2005, p. 174 sq.

13 Ludmila VLčKová, Benediktinska klášterní knihovna v Broumově (La bibliothèque de l'abbaye bénédictine de Broumov), Hradec Králové (Kruh), 1969, conclusion.

14 Petr Miкšíček, «Die Besiedlung der Grenzregionen », in: Petr Mikšíček, Matěj SpurnÝ, Ondřej Matějka, Susanne Spurná (dir.), Zmizelé Sudety (2006), Domažlice, Český les, 2007 (2éd. revue et augmentée), p. 95-101, ici p. 97.

15 Interview de Mme Růčková, maire de Braunau, Braunauer Rundbrief, n 4, 2004, p. 107. 
avoir été traduits en tchèque ${ }^{(16)}$. Aidés à partir de 1997 par le Fonds tchéco-allemand d'avenir, les Heimatkreise participèrent à des actions culturelles: pour le financement de tout ou partie des projets visant à conserver le patrimoine bâti, notamment les églises et les cimetières; pour entretenir le souvenir de la culture allemande. Ainsi existe-t-il aujourd'hui un prix littéraire de Broumov, décerné à des ouvrages en tchèque, en polonais et en allemand qui ont trait au petit pays. Le Heimatkreis de Braunau, qui a été chargé du choix de l'ouvrage en allemand ${ }^{(17)}$, a aussi agi pour faire aboutir l'accord de jumelage signé le 21 juillet 2002 entre Forchheim (Bavière) et Broumov. D’autres manifestations de coopération culturelle ont désormais lieu régulièrement, comme les concerts destinés à soutenir la restauration d'églises ${ }^{(18)}$. Depuis le milieu des années 2000, à côté d'associations de plus en plus nombreuses qui se consacrent à des projets très locaux comme la restauration d'un bunker, les pouvoirs publics sont beaucoup plus présents qu'avant pour dynamiser, coordonner et valoriser selon les méthodes scientifiques en vigueur le patrimoine (bâti notamment) encore existant. L'ensemble baroque de Broumov connaît désormais une ambitieuse campagne de restauration menée par Martin Mádl de l'Institut d'histoire de l'art à l'Académie tchèque des sciences, tandis que le musée de Hradec Králové vient de lancer un projet centré autour des industriels de la fin du XIX ${ }^{\mathrm{e}}$ siècle qui ont profondément influencé l'économie, la société et donc le paysage de la région ${ }^{(19)}$.

Pour nombre d'associations fondées après la révolution de 1989, le contact avec les anciens habitants expulsés était une condition nécessaire pour lever le voile sur le passé récent, tu par les autorités et très peu traité par l'historiographie. Les efforts se concentrèrent sur le point douloureux de l'expulsion; ainsi en 2002, les associations INEX et "Tuž se, Broumovsko » (traduit au mieux par "Bouge-toi, pays de Broumov », devise calquée sur celle du mouvement de gymnastique tchèque des Sokols) s'associèrentelles au maire de Wekelsdorf/Teplice nad Metuji, Věra Vitová, pour ériger, avec un courage politique certain, un monument à la mémoire des personnes assassinées en 1945 au lieu-dit Buchenberg/Buková hora ${ }^{(20)}$.

16 Par exemple par l'agence de voyage Neumann à Broumov qui a vendu plus de "livres des villages » par an que le Heimatkreis, bien que les ouvrages soient en allemand (Braunauer Rundbrief, $\mathrm{n}^{\circ}$ 5, 2001, p. 136).

$17 B R, \mathrm{n}^{\circ} 5,2001$, p. $138-139$.

18 C'est devenu une tradition de soutenir la restauration des églises baroques du pays de Broumov par des concerts (de l'association «Pro Arte antiqua» de Brno) et des opérations portes ouvertes, celle de 2015 se faisant par exemple en coopération avec l'Université de Wrocław, l'Institut d'histoire de l'art de l'Académie tchèque des sciences et l'association locale Omnium.

19 Je remercie Madame Machková, directrice du musée, de m'avoir transmis un exemplaire de ce projet destiné à montrer les caractéristiques communes de la région de Hradec Králové, formée en 2008 à partir de divisions administratives plus anciennes.

20 Cette " croix de la réconciliation ", réalisée par le sculpteur Petr Honzátko, a été conçue comme "symbole de la renonciation à la violence "; elle fait partie d'un ensemble qui, avec une autre sculpture et une allée plantée d'arbres, constitue un monument dédié au rapprochement des peuples. Elle a été financée en grande partie par le Fonds tchéco-allemand pour l'avenir (constitué par les deux États après la Déclaration de bon voisinage de 1997) pour 350000 couronnes, mais aussi par des entreprises locales et par le Heimatkreis de Braunau (pour 30000 couronnes, soit environ mille euros). Inaugurée le 15 septembre (jour du patrimoine culturel européen) avec de nombreuses personnalités (l'évêque 
Le déficit de connaissances historiques est progressivement comblé par les publications scientifiques. Depuis la fin des années 1990, les annales pluriannuelles des archives de Trutnov et de Náchod ${ }^{(21)}$ se font l'écho des recherches, universitaires ou non, menées sur leur district (Broumov appartenant à celui de Náchod), ajoutant à leurs domaines traditionnels (l'histoire de l'industrie textile ${ }^{(22)}$ et l'histoire locale médiévale et moderne) de nouvelles contributions d'histoire contemporaine, rendant par exemple publiques des sources jusqu'alors peu valorisées comme les archives photographiques privées ${ }^{(23)}$ ou les témoignages. Ces sources non imprimées sont utilisées avec le recul scientifique nécessaire, comparées systématiquement aux autres informations disponibles et exploitées par un nombre croissant de publications scientifiques (y compris par les musées et les universités); on peut donc en conclure que la patrimonialisation de l'héritage historique est en bonne voie, qui constitue à partir du passé, tel que ses traces nous permettent de le reconstituer, un héritage pour tous les habitants actuels, quelle que soit leur origine, tout en respectant les impératifs de conservation et de gestion participative des sources qu'implique une démarche vraiment patrimoniale.

\section{Retrouver l'histoire - se confronter à son histoire}

C'est au croisement de ce renouvellement de l'historiographie et du développement durable régional que se place l'association patrimoniale tchèque la plus connue, Antikomplex. Cette association suprarégionale fut fondée en 1998 par quelques étudiants ${ }^{(24)}$ d'après l'idée qu' « une histoire refoulée est la source de complexes non seulement chez l'individu, mais aussi au niveau de la société " ${ }^{(25)}$. S'appuyant explicitement sur le savoir accumulé par les anciens habitants allemands, elle ouvrit en 2002 une

Josef Kajnek de Pardubice, le président du Sénat Petr Pithart, les ambassadeurs d'Allemagne et de la République sud-africaine), des descendants des assassinés, une représentation du Heimatkreis et le président de la Sudetendeutsche Landsmannschaft, Bernd Posselt, cette initiative a reçu en 2003 le prix Franz-Werfel de la Fédération allemande des expulsés (Bund der Vertriebenen). Les lauréats sont l’ancien maire de Teplice Věra Vítová, de Petr Kulíšek (INEX) et de Jan Piňos («Tuž se, Broumovsko »). D’après Lenka Tvrdková (réd.), Vom Kreuz zur Versöhnung, Teplice, 2002, p. 98-104.

21 Stopami dějin Náchodska (Sur les traces de l'histoire de Náchod et sa région) et Ročenka státního okresního archivu $v$ Trutnově (Annales des archives du district de Trutnov), qui prend la suite de la revue Krkonoše-Podkrkonoší et de ses suppléments, lancée au milieu des années 1960 pour faire le lien entre musées, archivistes, érudits locaux et enseignants.

22 Depuis 1975 a été publiée une série de travaux sur l'entreprise Texlen de Trutnov et les entreprises d'avant 1948; signalons aussi E. BouzA et V. VAŇKová, Osm století textilní výroby na Broumovsku (Huit siècles de fabrication du textile dans le pays de Broumov), Hradec Králové, 1972.

23 C'est ainsi qu'Alexandra Teodoridisová a exploité des photographies privées de la fin de la guerre (libération, expulsion des Allemands) pour son mémoire de master à l'université d'Opava, matière d'un long article dans Ročenka státního okresního archivu v Trutnově, 1999: "Německá menšina na Královédvorsku po druhé světové válce » (La minorité allemande de la région de Dvůr Králové/ Königinhof après la Seconde Guerre mondiale), p. 47-129.

24 Petra Stuiber, "Suche nach verschwundenen Landschaften », Der Standard, 3 mars 2009. Voir aussi la thèse de Paul BAUER, "Les espaces frontaliers de la Bohême au XX⿳亠丷厂 siècle. Un essai de géographie de la mémoire », EHESS, 2010. Plusieurs de ces étudiants (par exemple Ondřej Matějka et Matěj Spurný) sont entre-temps devenus des spécialistes reconnus par le monde universitaire.

25 Source: association Antikomplex, «Pro média » (Questions fréquemment posées) [en ligne à l'adresse] www.antikomplex.cz/pro-media.html, consulté le 27 juillet 2015. 
exposition itinérante de photographies sur les confins, intitulée Zmizelé Sudety («les Sudètes, une région disparue ") où l'état actuel de ces territoires était comparé à celui d'avant 1938. Son catalogue bilingue tchèque-allemand paru en 2006 connut un tel succès (18000 exemplaires vendus en 2009) qu'il est devenu un élément incontournable de la réflexion sur le patrimoine des confins. L'objectif des auteurs était, en assumant l'histoire telle qu'elle s'était déroulée, de changer le regard sur ces territoires:

«S'il faut affirmer que notre engagement est dirigé contre quelque chose, alors disons qu'il se bat contre la disparition d'une perception vraiment attentive à notre environnement. Nous ne remarquons souvent même pas le manque d'harmonie ou la décrépitude des choses qui nous entourent. Nos sens peuvent s'adapter à presque tout, y compris à un contexte hostile. Mais si nous étions en mesure de contempler notre environnement avec des yeux tout neufs, nous verrions brusquement la chapelle détruite, l'étang envahi d'herbe et les tas d'immondices sur nos places de village [...]. Se dessine alors une mission: comment ces constatations dérangeantes sur la région frontalière peuvent aboutir à la naissance de quelque chose de neuf, d'intéressant, afin que le "pays sudète" ne soit plus un mot brûlant, mais qu'il revête une nouvelle signification, qu'il devienne le symbole d'un nouveau départ dans ce qui auparavant n'était associé qu’à la perte et à la décrépitude? " (26)

Pour Antikomplex, les préoccupations culturelles et environnementales trouvent leur traduction dans le paysage: c'est donc lui qui doit retenir toute l'attention. Plusieurs des associations de Bohême orientale partagent cette préoccupation et agissent par des corrections symboliques, comme la restauration des calvaires ou la réouverture de chemins. Il s'agit ainsi de lutter contre le déprimant sentiment de vide qui guette les habitants, «des hommes sans racines, plus habitués à survivre qu'à construire. Ce sont ceux-là qui vinrent après la guerre dans le paysage vidé de ses habitants, dans lequel il n'y avait rien sur quoi s'appuyer, rien qui leur permettrait de s'orienter, à eux qui étaient souvent perdus. Ici, il ne restait rien que le vide: vide de l'esprit, maisons vides. C'est à ce vide qu'ils se sont adaptés " ${ }^{(27)}$.

26 «Wenn man überhaupt davon sprechen kann, dass unser Engagement gegen etwas gerichtet ist, dann wendet es sich gegen den Verlust einer wirklich aufmerksamen Wahrnehmung unserer Umwelt. Die fehlende Harmonie oder den Verfall in unserer Umgebung bemerken wir oftmals gar nicht. Unsere Sinne sind in der Lage, sich an fast jede Umgebung anzupassen, auch an ein sehr ungastliches Umfeld. Wenn wir jedoch in der Lage wären, von Zeit zu Zeit unsere Umwelt mit völlig neuen Augen zu betrachten, würden wird plötzlich die zerstörte Kapelle, den zugewachsenen Teich und den Müllhaufen auf unserem Dorfplatz sehen [...]. Schon bald zeichnen sich nämlich die Umrisse einer großen Aufgabe ab: Wie können all diese beunruhigenden Feststellungen über die Grenzregion umgewandelt werden, so dass etwas Neues, Interessantes entsteht, damit 'Sudetenland' nicht mehr nur ein Reizwort ist, sondern mit einem neuen Inhalt gefüllt werden kann, damit es zum positiven Symbol für einen Neuanfang an einem Ort wird, der für uns zuvor in erster Linie Verlust und Verfall verkörperte? » Petr MikšíčEk, Matěj SpurnÝ, Ondřej MatĚjka, Susanne SpurnÁ, «Über das Projekt 'Das verschwundene Sudetenland' », in: Zmizelé Sudety (note 14), p. 62-65, ici p. 63.

27 «[... Die nicht verwurzelten Menschen, Menschen, die es eher gewöhnt sind zu überleben als aufzubauen. Und solche kamen in den Jahrzehnten nach dem Krieg in die leergefegte Landschaft, in der nichts war, auf das sie sich stützen konnten, was ihnen, den oft Verirrten, helfen konnte sich zu orientieren. Es blieben hier nur geistige Leere und leere Häuser. Und so passten sie sich der Leere an. » Tomáš FeřTEK, «Die Fotografien sind das schmerzliche Gedächtnis des Ortes », in: Zmizelé Sudety (note 14), p. 68-69, ici p. 69. 
Cette patrimonialisation-là n'est pas un simple retour à de bonnes méthodes d'étude et de gestion après deux périodes de dictature qui ont falsifié l'histoire et empoisonné l'environnement. Donner une épaisseur aux lieux qui permette de mieux les comprendre est aussi un acte pour enrichir l'imaginaire, sur « la terre en poussière qui vole au vent [où] il suffit pourtant de creuser le substrat pour découvrir des trésors de mémoire opiniâtre " ${ }^{(28)}$. Cette démarche a été clairement assumée par l'association Antikomplex, au risque d'une certaine naïveté, celle de ne pas voir dans le paysage disparu les traces d'une exploitation intense, y compris dans le passé: par une agriculture visant avant tout la rentabilité, par un tourisme cherchant à attirer les porteurs de devises d'au-delà de la frontière (autrefois les Allemands, désormais les Polonais), par une industrie usant sans ménagement des ressources naturelles. C'est que son objectif est autre: c'est celui d'éviter (ou de sortir de) la culture de la pauvreté, celle qui empêche de prendre son destin en main, qui borne l'horizon aux choses connues et le temps à celui du présent ${ }^{(29)}$.

Paradoxalement, la démarche d'Antikomplex se trouve corroborée par le fait qu'après 1945, les expulsés adoptèrent exactement la même attitude envers les paysages perdus, les considérant comme une clef pour la régénération et la fortification intérieure. Ce n'est pas le moindre mérite de cette démarche tchèque que de permettre une plongée dans la «mentalité » des expulsés allemands après 1945 - celle-là même qui intéresse tant la recherche depuis que le «mythe " de leur intégration rapide à la société ouestallemande a été remis en question ${ }^{(30)}$.

\section{Restaurer la sensibilité au paysage pour régénérer les hommes}

Il n'est guère étonnant que la vision historique souvent défectueuse donnée par les publications d'expulsés ait conduit les historiens à les écarter comme dénuées de valeur, si ce n'est celle (négative) d'entretenir l'illusion d'un passé idéalisé. Or, " les Heimatbücher ne sont pas qu'une documentation historique, mais bien plutôt une forme spécifique d'écriture du souvenir. Cette littérature renseigne sur les états d'esprit des réfugiés et expulsés, et constitue [...] une source de premier plan, jusqu’à présent négligée pour l'histoire des mentalités » ${ }^{(31)}$. Nous entendons montrer ici comment le passé, tout particulièrement la mémoire des paysages, a pu aider les migrants allemands de Bohême orientale après leur départ forcé.

28 Simon Schama, Le paysage et la mémoire (Landscape and Memory, 1995), Paris, Seuil, 1999, p. 649.

29 Ivo T. BudiL, « Das Sudetenland als gesellschaftliche Herausforderung », in: Zmizelé Sudety (note 14), p. 73-75, ici p. 74, citant l'anthropologue Oscar Lewis.

30 Paul Lüttinger, "Der Mythos der schnellen Integration: eine empirische Untersuchung zur Integration der Vertriebenen und Flüchtlinge in der Bundesrepublik Deutschland bis 1971 », Zeitschrift für Soziologie, vol. 15, 1986, p. 20-36. Le thème a été récemment repris par Andreas Kossert, Kalte Heimat. Die Geschichte der deutschen Vertriebenen nach 1945, Munich, Siedler, 2008.

31 «Dementsprechend sind diese Heimatbücher nicht nur historische Dokumentation, sondern in noch höherem Maß eine spezifische Form von Erinnerungsliteratur. Sie gibt Auskunft über den Bewusstseinsstand der Flüchtlinge und Vertriebenen und stellt, wie Kessler vermerkt, eine herausragende, aber bislang noch kaum genutzte mentalitätsgeschichtliche Quelle dar. » M. BEER, «Das Heimatbuch als Schriftenklasse» (note 6), p. 17. 


\section{Les bulletins d'expulsés contre la pauvreté morale}

$S$ 'il est bien connu que des repères spatiaux aident la mémoire, voire représentent la condition indispensable d'une mémoire collective ${ }^{(32)}$, seuls les textes des acteurs euxmêmes peuvent nous permettre de comprendre comment a fonctionné cette mémoire et surtout, comment elle a pu servir de ressource morale. Parmi ces textes, nous avons choisi les périodiques: leur intérêt par rapport aux Heimatbücher tient à leur publication pendant une longue durée, la plus grande diversité de leurs auteurs et leur réaction au contexte. En outre, la répétition de certains thèmes montre la popularité de ces derniers parmi les lecteurs qui, dans la période étudiée (jusqu'au milieu des années cinquante), représentaient une proportion importante des anciens habitants du même lieu. Ils constituent donc une source particulièrement valable pour se plonger dans les représentations des expulsés.

Les publications que nous étudions eurent en commun d'être d'origine religieuse ${ }^{(33)}$. Ce fut l'ancien abbé bénédictin de Braunau, désormais à Rohr en Bavière, qui lança la « Lettre circulaire de Braunau » en 1948 tandis que d'anciens curés des paroisses du district de Trautenau entendaient aussi réconforter leurs ouailles dispersées après l'expulsion ${ }^{(34)}$. Cela n'a rien de surprenant, puisque seuls les ecclésiastiques étaient autorisés à rassembler des expulsés dans les zones d'occupation occidentales (leur action était du reste tolérée en zone d'occupation soviétique).

Dès l'année 1949, les deux publications que nous avons étudiées eurent des auteurs plus diversifiés, mais cette évolution est malaisée à reconstituer puisque les articles ne sont pas signés. Dans le ton et le choix des sujets, ils se rapprochèrent de la Sudetendeutsche Landsmannschaft (SL) en formation. En 1952 furent fondés sous son égide les Heimatkreise de Braunau, Trautenau et Hohenelbe, réunis dans une subdivision appelée " paysage », le " paysage des Monts des Géants » (Heimatliche Landschaft "Riesengebirge »), ce qui nous rappelle que le terme "s'applique [aussi] à une unité d'occupation humaine, une juridiction, en fait, tout autant qu'à un lieu agréable à dépeindre » ${ }^{(35)}$. Au même moment qu'il annonçait la fondation du Heimatkreis, l'abbé Procope ouvrit le $B R$ à ses dirigeants qui prirent le contrôle de la rédaction d'une partie du bulletin ${ }^{(36)}$, l'autre restant jusqu'à aujourd'hui assurée par les bénédictins. Quant à la $R G B$, le fait que le chef-lieu, Trautenau, n'eut jamais la place qui aurait dû lui revenir d'après son importance passée s'explique sans doute parce que la revue devint le fief des anciens habitants de Hohenelbe, dominant dans les instances de la SL ${ }^{(37)}$. À partir de ce moment-là, le ton de la $R G H$ et du $B R$ se rapprocha au point de devenir identique, tout comme leurs points de vue sur la question des expulsés et la politique

32 Maurice Halbwachs, La topographie légendaire des évangiles en Terre sainte, Paris, 1941.

33 Des sondages dans la bibliothèque de l'IVDE montrent que c'est un fait assez répandu.

34 Par exemple, Fasten-Seelsorgebrief an die katholische Pfarrgemeinde von Marschendorf, 1947; Rundbrief an die katholische Pfarrgemeinde Gross-Aupa, édité à Weilmünster, 1946-1949 (d'après R. Hemmerle, Heimat im Buch [note 4], p. 278-279). Le périodique prend sa forme définitive en 1958, par la fusion du Riesengebirgsheimat. Monatliches Heimatblatt für die ehemaligen Kreise Trautenau und Hohenelbe et de Aus Rübezahls Heimat.

35 S. Schama, Paysage et mémoire (note 28), p. 17, citant les termes de landschap (hollandais) et Landschaft (allemand).

$36 B R, \mathrm{n}^{\circ} 6,1952$, p. $4-5$.

37 Riesengebirgsheimat, janvier 1954, p. 18. 
(allemande comme étrangère) qui devinrent, sans surprise, ceux de la Landsmannschaft ${ }^{(38)}$. Mais cet alignement resta discret. Rien de ce qui fâchait ne fut abordé par ces feuilles visant d'abord à rassembler et les choix restèrent implicites: quasiment aucune allusion directe à la période nazie, peu d'exemples de réussite de compatriotes dans la «nouvelle patrie » (qui auraient pu susciter la jalousie), de rares et discrètes évocations des divergences politiques d'avant-guerre.

Pour beaucoup de lecteurs, l'expulsion avait été un traumatisme qui s'était ajouté à la défaite écrasante de l'Allemagne, à l'effondrement presque complet des cadres habituels de référence et aux destructions massives de la guerre totale. Le ton adopté par les périodiques fut, sans relâche, celui du réconfort, tout particulièrement jusqu'à la Loi de péréquation des charges (1952) et la Loi des expulsés (1953) en RFA (qui organisèrent un système particulier d'assistance et d'indemnisation à ceux qui avaient tout perdu par faits de guerre). À travers les lignes, on peut lire en creux le profond sentiment de dévalorisation éprouvé par les migrants forcés, prenant la forme chez certains d'une apathie complète, confirmée par de nombreuses sources. Les deux périodiques eurent donc pour objet principal d'aider leurs lecteurs dans l'épreuve, non seulement en les informant sur les dispositions législatives à leur avantage, mais encore en les encourageant et en les aidant à faire le deuil de la patrie perdue (sans exclure l'idée de retour, mais dans la pleine conscience qu'il importait de se rebâtir une existence "dans la nouvelle patrie » de la RFA et que les modifications apportées au peuplement de la Bohême en 1945 ne seraient pas aisément réversibles). Chaque numéro consacra entre une et trois pages au soutien moral et spirituel, tant en montrant l'intérêt de l'opinion mondiale pour la question des réfugiés ${ }^{(39)}$ que par des incitations à faire preuve de force morale et des «vertus allemandes » d'ordre et de travail prouvées par les biens culturels laissés dans les territoires perdus ${ }^{(40)}$. En effet, les encouragements s'appuyèrent sur les performances passées: monuments de la Heimat, grands hommes et réalisations économiques furent chargés de rappeler le potentiel créatif et entrepreneurial du groupe prolétarisé, dans un langage qui perdit progressivement (dans les années soixante-dix) ses connotations racialisantes. L'aide au deuil se traduisit par des rubriques régulières: des poèmes, souvent en dialecte, qui déploraient la patrie perdue; le rappel des joies vécues en commun (fêtes d'autrefois ou rassemblements contemporains); des éditoriaux au ton invariablement optimiste, montrant dans les

38 Ceci rejoint l'observation de Radomír Luža, historien tchèque en exil et auteur d'un des premiers ouvrages d'après-guerre sur l'expulsion des Allemands, qui notait en 1964 l'étonnante similitude des associations sudètes (SL, Ackermann-Gemeinde, Adalbert-Stifter-Verein et, à l'époque, Collegium Carolinum, enfin l'Arbeitsgemeinschaft zur Wahrung sudetendeutscher Interessen) quant à leur vision de l'histoire. Cité par T. WEgER, 'Volkstumskampf' (note 11), p. 328.

39 Le soutien du pape Pie XII qui, dès Noël 1945, avait publiquement compati au sort des expulsés allemands, fut particulièrement mis en relief. Sur cette question, voir Michael Hirschfeld, Katholisches Milieu und Vertriebene: eine Fallstudie am Beispiel des Oldenburger Landes, 1945-1965, Vienne/Cologne/Weimar, Böhlau, 2002, p. 143 sq.

40 Tobias Weger a raison de voir dans ce code moral une continuité directe avec la définition racialisante des Allemands formée au cours du XIX ${ }^{\mathrm{e}}$ siècle (p. 333 sq.), mais on peut tout aussi remarquer, comme Jutta Faehnrich l'a fait dans son étude sur environ cinq cents Heimatbücher d'expulsés, que les vertus mises en avant sont celles exigées de n'importe quel travailleur immigré: ardeur au travail, docilité et endurance (Jutta FAEHNRICH, «Erinnerungskultur und Umgang mit Vertreibung in Heimatbüchern deutschsprachiger Vertriebener ", Zeitschrift für Ostmitteleuropa-Forschung, 2 [2003], p. 191-229, ici p. 222-223). 
premières pages un avenir ouvert par des exemples choisis dans le travail auprès des jeunes ou la réussite d'anciens habitants. Les dernières pages furent consacrées aux nouvelles de la diaspora données dans un ton familier, parfois proche du dialecte, illustrées de nombreuses photos des anciens voisins, des groupes locaux dispersés en Allemagne et en Autriche, pour les avis de mariage ou de décès.

\section{À la recherche $d u$ genius loci}

Il n'est donc guère surprenant que le terme de Heimat occupe une place centrale dans ces pages. Nous allons nous pencher ici sur sa modalité particulière qu'est le paysage de la patrie d'origine, ce qui va nous permettre de modifier quelque peu la conception habituelle de cette notion de Heimat, que l'historiographie des expulsés présente de façon souvent figée.

Constatons d'abord que les évocations des paysages perdus connurent un succès durable auprès des lecteurs expulsés de Bohême orientale. Jusqu'aujourd'hui, de nombreux numéros des bulletins que nous avons étudiés, parfois chaque numéro d'une même année, présentent un ou plusieurs itinéraires à travers la région d’origine, jadis fondés sur des souvenirs puis, depuis les années soixante-dix, sur des excursions contemporaines. En effet, la facilité grandissante des voyages en Tchécoslovaquie à cette époque-là poussa explicitement les responsables du Heimatkreis de Braunau à envisager un autre type de publication, apte à servir de guide dans la Heimat, y compris à ceux qui n'y étaient pas nés ${ }^{(41)}$ : ce fut l'origine des Heimatbücher.

Dans les périodiques antérieurs qui nous intéressent ici, les guides de promenade pouvaient se présenter d'une façon relativement neutre, comme cet extrait de la $R G H$ d'août 1954; mais les passages en italiques (soulignés par nous) montrent qu'il repose sur des conceptions qui ne sont pas indifférentes.

«L'une des plus jolies promenades conduit au café de la Hutte à travers la vallée d'Arnsdorf, restée épargnée par l'industrie. Même si la route qui longe souvent le ruisseau Seigen vaut déjà la peine, lorsque les merisiers sont en fleur, nous préférons passer par les prés pour éviter d'être gênés par la poussière et le passage des voitures [...]. Qui ne craint pas les montées et veut jouir d'un magnifique panorama sur les montagnes choisisse de passer par le Töpferberg sur un chemin balisé durant deux bonnes heures. On prend à droite de la route et après une montée courte, abrupte, on atteint les hauteurs. Au-dessus de la forêt, la vue sur la Montagne noire est magnifique. On aperçoit aussi les deux églises de Herrmannseifen, celle de Mohren, le coteau de Ladig, le chalet Hoffmann et la vallée Noire à gauche. Après les maisons du Töpferberg, qu’on dit maintenant inhabitées, on arrive à la chapelle Sainte-Anne d'où l'on embrasse tout le paysage d'Arnau et, en passant le long du réservoir d'eau municipal, on atteint la route de la montagne ${ }^{(42)}$.

41 Ainsi, «cet abrégé est intéressant pour tous les compatriotes qui souhaitent se faire une impression [de Großdorf] [...] notamment pour les membres de la génération née après [l'expulsion] qui souhaitent se préparer intellectuellement à un voyage au pays de leurs aïeux » («Der Abriß aber [ist] durchaus für alle Landsleute von Interesse, die sich einen raschen Überblick verschaffen möchten. Daher gewinnt dieser erste Hauptabschnitt seine Bedeutung, insbesondere für Angehörige der nachgeborenen Generation, die eine Fahrt in die Heimat ihrer Vorfahren gedanklich vorbereiten möchten »). Préface d'Erhard Meissner au Heimatbuch Die Gemeinde Großdorf de Herbert Kinzel, Forchheim, 1999, p. 7.

42 «Einer der schönsten Spaziergänge führt durch das von der Industrie noch verschonte Arnsdorfer Tal nach der Hüttenschenke. Wenn schon der Weg auf der Straße oft längs des Seigenbaches, namentlich 
Cette description, à première vue neutre, appartient à la tradition du guide de randonnée qui, depuis le XIX ${ }^{\mathrm{e}}$ siècle, est considérée à travers l'Europe comme le meilleur moyen pour retrouver à moindres frais une nature intacte, «épargnée par l'industrie», dont le guide se porte garant qu'elle produira une impression esthétique («l'une des plus jolies promenades »). Loisir accessible à tous ou presque, la randonnée a connu un vif succès dans les sociétés en voie d'industrialisation, liée même dans les milieux modestes à l'intérêt pour la botanique, l'observation de la faune locale et le désir de se ressourcer, sans parler de la peinture de paysage. Les promoteurs des Monts des Géants et, dans le pays de Braunau, des rochers d'Adersbach l'ont développée à la fin du XIX ${ }^{\mathrm{e}}$ siècle.

Rien de surprenant dans ce texte donc, si ce n'est qu'il a été rédigé après l'expulsion. Ainsi les maisons du Töpferberg sont-elles réputées abandonnées de leurs habitants: le rédacteur ne saurait aller vérifier lui-même. En outre, il n'est pas impossible que leurs habitants en aient été chassés s'ils étaient allemands. Le présent de narration est un moyen stylistique pour supprimer la distance et rendre possible, par la pensée, une activité impossible en 1954. Ce rappel de plaisirs esthétiques est, nous en faisons l'hypothèse, au service de la reconstruction de soi que les journaux d'expulsés ont entreprise auprès de leurs lecteurs démoralisés par l'expulsion.

Très souvent, ce qui a trait à la Heimat a été considéré comme la clef de la mémoire collective des expulsés: ces évocations formeraient, d'après Jutta Faehnrich, les « souvenirs canoniques » du groupe ${ }^{(43)}$. Sous la plume des chercheurs allemands ${ }^{(44)}$, ces souvenirs prennent souvent le caractère d'un refuge contre le présent, donnent une impression générale d'immobilité qui réduit la Heimat à une idylle rétrograde. Mais ici, la variante de la Heimat qu'est le paysage prend un sens nettement plus dynamique, comparable à l'usage qui est fait aujourd'hui de la notion antique de genius loci par les acteurs tchèques du patrimoine, chez qui elle est omniprésente. Cette métaphore ${ }^{(45)}$ permet de désigner le caractère particulier de lieux dignes d'être restaurés et protégés; en même temps, comme sa traduction par « esprit du lieu » l'indique, elle contient une

im Frühjahr, wenn an den Ufern die Traubenkirschen blühen, sich lohnt, so können wir doch abseits von der Straße auf Wiesenwegen ohne vom Staub und dem Rasen der Autos belästigt zu werden, dasselbe Ziel erreichen. [...] Wer etwas Bergsteigen nicht scheut und einen herrlichen Ausblick auf das Gebirge genießen will, wählt den markierten Weg über den Töpferberg, der reichlich zwei Stunden dauert. Man biegt rechts von der Straße ab und nach einem kurzen, steilen Anstieg hat man die Höhe erreicht. Oberhalb des Waldes genießt man eine herrliche Aussicht auf den Schwarzen Berg. Man sieht auch die beiden Kirchen von Herrmannseifen, die Kirche von Mohren, die Ladighöhe, die Hoffmannsbaude und links Schwarzental. Über die Töpferberghäuser, die jetzt unbewohnt sein sollen, gelangt man zur Annakapelle, von wo man einen herrlichen Ausblick auf Arnau genießt und an dem Reservoir der städtischen Wasserleitung vorbei erreicht man die Gebirgsstraße. »Riesengebirgsheimat, août 1954, p. 3.

43 J. FAEHNRICH, « Erinnerungskultur» (note 40), p. 194.

44 Voir par exemple Wolfgang Kessler dans « Heimatbücher zum historischen Nordostdeutschland », qui évoque les fonctions du recours à la Heimat - orientation, sentiment, souvenir - sans entrer plus avant dans l'analyse des émotions. Dans: M. BeEr (dir.), Das Heimatbuch (note 6), p. 101-127, ici p. 115.

45 Qui, rappelons-le, désigne la personnalité (d'un homme, d'un État, d’une chose, d'un lieu...) et notamment ce qui en est perçu par les sens, ce qui permet aussi d'exister vraiment; un terme qui s'applique aussi bien aux êtres qu'aux lieux et aux choses, comme le livre: victurus genium debet habere liber (MARTial, 6, 60, 10). 
allusion magique ou religieuse, une promesse de transformation qui se retrouve aussi dans la sensibilité à la nature dont les pages que nous avons lues témoignent.

\section{Performativité de la mémoire des lieux}

C'est en se replongeant dans une relation harmonieuse et durable entre l'homme et son environnement que les expulsés déracinés parviendront à refaire leurs forces; à retrouver, en réactivant leurs sens, l'accès à une dimension morale supérieure qui les réconfortera. L'extrait suivant du même périodique en témoigne:

"Août, mois béni et mois d'abondance dans notre petite patrie, qui ne se souvient volontiers de toi! Quand ici dans la nouvelle patrie nous allons à travers champs, il nous semble que c'est l'automne et nous avons froid même au plus chaud du jour. Ce sont que chaumes qui s'étendent aussi loin que porte le regard, jusqu'à l'horizon.

[La narratrice passe aux forêts de son enfance]. Le mois d'août nous faisait encore d'autres dons. La forêt familière et les hauteurs nous attendaient pour nous combler. En bas, les myrtilles avaient déjà été grappillées. Mais si l'on montait, elles mûrissaient seulement en août, si grosses, si sucrées qu'on croyait ne rien avoir jamais goûté de meilleur. Il y avait tant de framboises et de mûres dans les coupes forestières que chaque ménage pouvait en tirer suffisamment de jus de framboise pour passer l'hiver [...].

La plupart du temps, on allait tôt aux champignons. Surpris, les oiseaux s'éveillaient et sautillaient pour nous détourner de leurs petits. Le soleil se levait, la forêt était encore sombre comme une église. De la vallée montait la cloche du matin comme si elle franchissait dans l'air les degrés de Dieu; les [?], buissons et mousses, pleins de rosée, nous mouillaient les pieds, mais cela faisait partie de la promenade. Nous étions pleins de joie, de la joie qui naît d'un nouveau jour et de la certitude de recevoir un cadeau. La forêt nous en a toujours fait. Nous ne sommes jamais rentrés les mains vides, même si le mouchoir noué en baluchon n'était parfois pas bien plein et pendait mollement. Un petit bouquet de bruyère, quelques étoiles d'arnica, peut-être même une tige de lys martagon, de pied-d'alouette ou de gentiane auraient à eux seuls valu la peine de venir vivre un nouveau matin, dans la forêt et sur les montagnes de chez nous ${ }^{(46)}$.

46 Olga Braunauer, "Gabenmonat August », même numéro de la RGH, p. 2: "August, du segen- und gabenreicher Monat in der Heimat, wer erinnert sich nicht gerne deiner! Wenn wir hier in der neuen Heimat im August über die Felder gehen, dann herbestellt es und fröstelt uns auch in der größten Hitze. Es sind die Stoppelfelder, die sich, soweit das Auge reicht, bis zum Horizont hin dehnen [...]. Der August war noch in anderer Hinsicht ein schenkender Monat. Der heimatliche Wald und die Berghöhen warteten darauf, uns reichlich zu versorgen. Die Schwarzbeeren waren wohl in den tieferen Lagen schon abgeklaubt. Wenn man aber höher hinauf kam, reiften sie erst im August, und waren so groß, so zuckersüß, daß man glaubt, in der Welt nichts Besseres verkostet zu haben. Himbeeren und Brombeeren gab es in den Waldschlägen so [unleserlich: sehr?], daß jeder Haushalt seinen Bedarf an Himbeersaft für den ganzen Winter decken konnte. [...] Zumeist ging man schon zeitig früh in die Pilze. Die Vögel erwachten und huschten aufgescheucht, um ihre Jungen besorgt, umher. Die Sonne war erst im Aufgehen, der Wald war noch dunkel wie eine Kirche. Aus dem Tale kam der Morgenläuten wie auf Gottes Stufen heraufgeschwebt, Schmehlen [?], Sträucher und Moos trieften von Tau und durchnässten uns zwar, aber das gehörte irgendwie dazu. Nur Freude war in uns, die Freude an einem neuen Tag und jenes schöne Gefühl, wenn man weiß, daß man ein Geschenk erhält. Beschenkt hat uns der Wald immer. Nie ließ er uns leer nach Hause gehen, auch wenn einmal der Pinkel nicht so straff gefüllt war, sondern schlapp hernieder hing. Ein Sträußlein Erika, einige große Arnikasterne, vielleicht sogar ein Stengel Türkenbund oder Rittersporn oder Enzian wären allein schon wert gewesen, das Erwachen eines neuen Morgens im heimatlichen Wald und auf den Bergen in der Heimat zu erleben. » 
Un souvenir d'enfance, aux notations sentimentales (or le sentiment naît lorsqu'on est conscient de la perte de l'objet aimé) et au ton discrètement pathétique (la perte est irrémédiable) : nous sommes indubitablement dans une évocation de paysage de style romantique, sans grande originalité, mais qui a dû toucher ses lecteurs, puisque ce type de récit se répète au cours des années dans les périodiques étudiés. Ce qui distingue ici le paysage de la patrie d'origine, par rapport à celui de la terre certes d'accueil, mais si étrangère, c'est le souvenir d'une harmonie exceptionnelle entre l'homme et la nature. Cette dernière, notons-le, n'a ici pas de coloration nationale: si l'on peut présumer qu'avant 1938, l'auteure aurait glosé sur le caractère typiquement allemand de la forêt, le terme n'apparaît pas en 1954 et pour cause. Désormais, la narratrice se trouve en Allemagne (" die neue Heimat: la nouvelle patrie, le nouveau chez-soi »), mais ne s'y sent pas chez elle. La topographie jusqu'alors « établie, élaborée et enrichie » dans le discours nationaliste sudète pour conférer à l'identité nationale la "féroce mystique » d'une tradition de paysage spécifique est ici adoucie pour revenir à la tradition de la forêt cathédrale, qui nous permet de trouver consolation à notre mortalité ${ }^{(47)}$. La communauté qui a été perdue est celle de la famille, le village, en somme l'environnement familier. À travers les lieux communs auquel il puise, comme celui de la forêt-cathédrale, ce texte rappelle aussi la richesse de la tradition paysagère (européenne) dans laquelle nature et culture ne s'excluent pas. Au contact de la nature peut se réactiver le « trésor de mémoire opiniâtre » qui se trouve dans chaque paysage européen, garantie de richesse intérieure ${ }^{(48)}$.

Le patrimoine dont il est question ici est uniquement idéel; il n'en est pas moins présenté dans nos sources comme un ressourcement pour une population prolétarisée, guettée par le découragement. En effet, la réunion des trois dimensions suivantes fait que ce paysage purement mémoriel procure un sentiment d'unité intérieure: le plaisir esthétique; la dimension communautaire (cet environnement a un sens pour la communauté, il est donc à préserver, ne serait-ce que par le souvenir); enfin, la dimension spirituelle, car il donne accès à une dimension morale supérieure, à la divinité. En l'absence de tout texte théorique dans nos sources, nous empruntons cette analyse au poète Wordsworth, l'un des plus fins descripteurs du paysage sentimental romantique. Au début de l'âge industriel, il influença profondément ses contemporains ainsi que la génération suivante. L'art des jardins et la peinture de paysage au XVIII ${ }^{\mathrm{e}}$ siècle avaient déjà formé le regard des Anglais cultivés à chercher des éléments évoquant le pittoresque et le sublime dans certains lieux naturels, intérêt renforcé par la théologie naturelle (la contemplation de la nature, œuvre du dessein divin, venant compléter l'étude de la révélation christique). C'était au même moment que l'industrialisation commençait dans les Midlands; elle était suffisamment avancée dès 1820 pour montrer qu'elle infligeait d'irréparables modifications à l'environnement; des dommages dont les conséquences étaient esthétiques et morales, en ce que l'urbanisation, l'uniformisation des modes de vie et la recherche du profit à court terme, y compris à la campagne, détruisaient les particularités, la variété et l'impression d'authenticité que les paysages anciennement humanisés présentaient. En conséquence, il devenait impossible à

47 Les termes entre guillemets sont empruntés à S. Sch AmA, Paysage et mémoire (note 28), p. 21. 
l'homme de se régénérer dans un paysage dénaturé, planté de pins à croissance rapide et semé de constructions à bon marché( ${ }^{(4)}$.

Wordsworth, rejoint par d'autres poètes comme Coleridge, ravis par l'harmonie délicate du Lake district dans lequel le poète s'était établi en 1799, réagit en donnant à la poésie la tâche de « restaurer ou d'accroître les capacités d'appréciation esthétique de la nature, puis de révéler les possibilités d'une coexistence harmonieuse entre l'homme et son environnement naturel " ${ }^{(50)}$, notamment par son Guide to the Lakes de 1810, qui soulignait que dans la région des Lacs au nord de l'Angleterre, l'action anthropique ne perturbait en rien les équilibres naturels, « ce qui en fait un patrimoine - notamment paysager - à protéger ${ }^{(51)}$. Son guide visait à former le regard de ses contemporains; mettant en mots dans son œuvre le sentiment profond d'être chez soi qu'il éprouvait au bord des Lacs, il employa son talent à enseigner à ses contemporains à ré-apprécier la beauté du monde pour, "sans le recours à des stimulants violents et grossiers ", la voir de façon à en être touché par toutes les fibres de son être. Pour lui, à condition d'aborder la nature dans une indispensable disponibilité spirituelle, c'est en conjoignant l'éducation du regard et l'émotion spontanée devant les beautés naturelles qu'il était possible de voir la complexité du monde et d'en ressentir l'infinie charge émotive ${ }^{(52)}$.

\section{Conclusion}

Nous retrouvons ici les deux mouvements que nous avons décrits précédemment, où le paysage n'apparaît pas ici seulement comme une forme culturelle parmi d'autres, mais bien comme un moyen thérapeutique. En effet, les thuriféraires des paysages de Bohême orientale veulent lutter contre un sentiment délétère de déracinement (" de vide ») et d'impuissance par rapport à l'environnement. Leur but est la régénération des individus et des groupes sociaux; il s'agit de (re)créer un lien affectif entre l'homme et son environnement, d'une façon qui se veut démocratique et accessible à tous. Le mouvement plus proche de nous dans le temps, celui de la patrimonialisation dans l'actuelle République tchèque, s'est donné pour but de refaire du lien social et de restaurer les capacités sensibles des habitants par l'infini pouvoir émotif des paysages, à condition qu'on veuille bien porter « un regard neuf» sur eux. Lautre, celui des bulletins des expulsés, est resté à usage communautaire, mais comptait sur les mêmes ressources pour permettre aux expulsés de trouver une place dans leur société d'accueil.

Le détour par Wordsworth nous a donc permis de découvrir la proximité inattendue de ces concepts, employés dans des situations où s'est imposé le sentiment d'une rupture violente avec l'environnement. La Heimat pour les uns, le genius loci pour les autres cherchent à retrouver à la fois "le lieu (environnement naturel) et le lien (social) », « en un moment [où] le lieu fait lien » ${ }^{(53)}$. En cela, notre étude enrichit l'approche historiographique du Kulturlandschaft, considéré habituellement sous l'angle de la construction nationale (et nationaliste) des groupes européens depuis la fin du

49 Ch.-F. Mathis, In Nature We Trust (note 1), p. 103-104.

50 Ibid., p. 97-98.

51 Ibid., p. 99.

52 Ibid., p. 98 et 100.

53 Propos empruntés à Michel Maffesoli, Philosopher en français, Paris, PUF (Quadrige), 2001, p. 431. 
$\mathrm{XIX}^{\mathrm{e}}$ siècle. La parenté évidente des conceptions que nous avons présentées ici tient en effet à une approche commune du paysage patrimonialisé (celui qui a suffisamment de valeur aux yeux de ses habitants pour être préservé) qui ne met pas la définition nationale au premier plan. Cette approche non nationale rend possible non seulement de mieux comprendre l'état d'esprit des expulsés allemands à leur arrivée en Allemagne, mais aussi d'envisager qu'au-delà des vicissitudes du $\mathrm{XX}^{\mathrm{e}}$ siècle, les sociétés européennes partagent le même héritage d'une riche tradition paysagère dans laquelle nature et culture ne s'excluent pas.

\title{
Résumé
}

Notre terrain d'étude, l'extrémité orientale du district de Hradec Králové (République tchèque), a connu deux mouvements de patrimonialisation successifs depuis la Seconde Guerre mondiale, par les anciens habitants germanophones expulsés après 1945 puis par des associations locales tchèques qui cherchent aujourd'hui à augmenter l'attractivité de leur région. Il leur arrive d'ailleurs de collaborer; mais plus fondamentalement, leur démarche est d'une étonnante similitude en ce qui concerne la valeur accordée au paysage. L'analyse de leurs publications montre en effet que le paysage, décrit dans les études sur le nationalisme en Europe centrale comme un élément majeur pour construire la nation, est ici conçu au premier chef comme une ressource morale, apte à refaire du lien social (par exemple en y réparant les chemins), comme à restaurer la capacité sensible de ses visiteurs, donc l'estime de soi et la capacité à se projeter dans un avenir durable. Ceci rejoint de manière étonnante les préoccupations exprimée en Angleterre dès la première moitié du XIX ${ }^{e}$ siècle à propos du paysage menacé par l'action de l'homme - ici, l'industrialisation - et les efforts faits pour conserver certains paysages remarquables comme résultat d'une interaction séculaire entre l'homme et son environnement. Par-delà les différences de période et de contexte, on voit donc ici à l'œuvre une même conception européenne du paysage, certes romantique, mais qui n'est pas nationale.

\begin{abstract}
We studied two attempts to define and preserve the worthwhile past of a small region between the Sudeten mountains and Silesia in the Eastern part of the Czech republic: first by the expelled German-speaking inhabitants collecting memories in exile after 1945, then by Czech associations founded in the aftermath of the "Velvet revolution." Both show an amazing similarity in their conception of the landscape as a therapeutic resource for individuals and for local society, harmed by war, expulsion or a ruthless dictatorship disregarding human rights and environment. They both aim at restoring the ties of the former or actual inhabitants with the outstanding beauty of the local landscape, either by publishing imaginary walks through the region that had just expelled them or by preserving nature and historical buildings. Hence humans and society seek a cure for their feeling of estrangement, which could lead to despair, lack of self-confidence and inability to imagine a sustainable future. This conception reminds us of the first European attempt to protect an endangered landscape, the English Lake district movement in the first half of the nineteenth century, which shows that the celebration of natural scenery in Central Europe, though no doubt romantic, is not necessarily an attempt to nationalize nature.
\end{abstract}

